\title{
When does ambiguity fade away?
}

\author{
Filippo Massari* \\ University of East Anglia \\ \& Bocconi University
}

Jonathan Newton

Kyoto University

July 11, 2020

\begin{abstract}
If the prior support is finite, long-run ambiguity is known to be a possible outcome only if the learning problem is misspecified (Marinacci and Massari, 2019). We show that if the prior support is naturally rich, long-run ambiguity cannot occur.

Keywords: Ambiguity, learning, robust statistical decisions, misspecified learning.

JEL Classification: D81, D83, C11
\end{abstract}

\section{Introduction}

Researchers have considered the implications of ambiguity for many economic phenomena. Examples include trade (Kajii and Ui, 2006), portfolio selection (Garlappi et al., 2006), risk pricing (Augustin and Izhakian, 2020), savings behavior (Hansen et al., 1999), job search (Nishimura and Ozaki, 2004) and the possibility of speculative bubbles (Werner, 2019). ${ }^{1}$ Given the salience of ambiguity in economic and financial research, it is natural to wonder about how persistent it is. In the current paper, we focus on the multiple prior model of ambiguity and consider conditions under which ambiguity fades away in the long run as a consequence of learning.

When a Bayesian decision-maker's set of priors comprises a finite set of iid models that includes the true model, Marinacci (2002) shows that ambiguity fades away over time as the decision-maker learns the true model. Marinacci and Massari (2019) drop the iid assumption and allow the problem to be misspecified so that it is impossible for the decision-maker to learn the true model. Nevertheless, they can still provide tight conditions under which ambiguity

${ }^{*}$ Corresponding author. E-mail: massari3141@gmail.com.

${ }^{1}$ The reader is referred to the survey article by Gilboa and Marinacci (2016) for more examples. 
fades away. However, many applications, including all those mentioned above, feature decisionmakers with sets of priors on the whole parameter space, a set of positive Lebesgue measure. It is this latter setup that we study in the current paper. We demonstrate that, under natural assumptions, ambiguity fades away on all sequences with finite maximum likelihood. Over time, all the posteriors concentrate on a shrinking neighborhood of this estimate and ambiguity fades away. Notably, the result holds even if the maximum likelihood estimate does not converge to a limit: all priors eventually concentrate around the estimate, even if the estimate itself changes over time.

The impact of ambiguity fading away will differ across models. For example (I) Kajii and Ui (2006) give necessary and sufficient conditions under which trade can take place under ambiguity. Trade that does take place in these conditions will be unaffected by ambiguity fading away, but additional opportunities for trade may arise. ${ }^{2}$ (II) Werner (2019) shows that speculative trading bubbles can arise when market participants have common but ambiguous beliefs. Consequently, if ambiguity fades away, then another explanation for long-run speculative trade is required. (III) Garlappi et al. (2006) consider mean-variance portfolio selection with an ambiguous parameter. If ambiguity fades away, then the model eventually returns to the classical mean-variance model (Markowitz, 1952; Sharpe, 1970). ${ }^{3}$

There are other models that study the effect that learning has on ambiguity, and some of these models (see, e.g. Epstein and Schneider, 2007) allow for persistent ambiguity. The multiple prior model we describe relies on the strong law of large numbers. Because the strong law of large numbers holds for each prior, all priors concentrate on the same model and ambiguity fades away.

\section{Probabilities}

We consider a family of models $\mathcal{M}=\left\{P_{\theta}: \theta \in \Theta\right\}$ parameterized by a positive Lebesgue measure parameter set $\Theta \subset \mathbb{R}^{k}$, defined on a $\sigma$-algebra $\Sigma^{\infty}$ of subsets of $X^{\infty}$ with representative element $x^{\infty}=x_{1}, x_{2}, \ldots$, where $X^{\infty}:=\times^{\infty} X$ is the infinite Cartesian product of a state space $X$ with representative element $x$ and $\sigma$-algebra $\Sigma$. With a slight abuse of notation, we use $P_{\theta}\left(x^{t}\right)$ to denote the probability that model $P_{\theta}$ attaches to the cylinder with base

\footnotetext{
${ }^{2}$ In the model of Kajii and Ui (2006), trade between two players is possible if and only if their sets of priors do not overlap. It is easy to see that if their sets of priors do not overlap under ambiguity, then the players will differ in their beliefs after ambiguity has faded away. Conversely, even if their sets of priors overlap under ambiguity, it is possible that the players will differ in their beliefs after ambiguity has faded away.

${ }^{3}$ Garlappi et al. (2006); Hansen et al. (1999) belong to a special class of ambiguous models known as $\varepsilon$ contamination models (see, e.g. Berger, 2013), in which the set of priors consists of all models within some distance $\varepsilon$ of an estimated model. Such models satisfy our condition of a positive Lebesgue measure of models in the support of the decision-maker.
} 
$x^{t}$ (i.e., $\left.C y l\left(x^{t}\right):=\left\{x_{1}, \ldots, x_{t}, X_{t+1}, X_{t+2}, \ldots\right\}\right)$, as well as the likelihood that model $P_{\theta}$ attaches to the partial sequence $\left(x_{1}, \ldots, x_{t}\right)$.

Specifically, we focus on the case in which $\mathcal{M}$ is a regular exponential family in the natural parametrization - most of the commonly used distributions form a regular exponential family and can be re-parametrized into their natural parametrization form (e.g. Gaussian, Multinomial, Poisson,...) - , which covers most standard learning settings, including those cited in the introduction. $^{4}$

Definition 1 (Exponential family.). Let $\nu$ be a $\sigma$-finite measure on the Borel subsets of $\mathbb{R}^{k}$ and $\mathcal{H}$ be the support of $\nu$. Define

$$
\Theta:=\left\{\theta \in \mathbb{R}^{k}: \int_{\mathcal{H}} \exp \left(\theta^{T} x\right) \nu(d x)<\infty\right\}
$$

define a function $\psi$ and a probability densities $P_{\theta}$ on $X$ with respect to $\nu$ by $\psi(\theta):=\ln \int_{X} \exp \left(\theta^{T} x\right) \nu(d x)$ and $P_{\theta}(x):=\exp \left(\theta^{T} x-\psi(\theta)\right)$. We refer to $\mathcal{M}:=\left\{P_{\theta}(x) \mid \theta \in \Theta\right\}$ as an exponential family in the natural parametrization. An exponential family is regular if $\Theta$ is an open set.

The prior information about the parameters is summarized by prior distributions $\mu \in \Delta \Theta$. The set of prior distributions is $\mathcal{C}$. For any prior distribution $\mu \in \mathcal{C}$ the joint distribution of the parameters and the observations is $P^{\mu} \in \Delta\left(\Theta \times X^{\infty}\right)$, defined by, for all sets $A \subseteq \Theta$ and all cylinders $x^{t}$

$$
P^{\mu}\left(A \times x^{t}\right):=\int_{A} P_{\theta}\left(x^{t}\right) d \mu .
$$

We denote by $\mu\left(. \mid x^{t}\right) \in \Delta \Theta$ the usual posterior given the observations $x^{t},{ }^{5}$ while $P^{\mu}\left(. \mid x^{t}\right) \in$ $\Delta(\Theta \times X)$ is the one-step-ahead predictive distribution of $x_{t+1}$, given observations $x^{t}$. By definition, for all $A \subseteq \Theta$ we have

$$
P^{\mu}\left(A \times x_{t+1} \mid x^{t}\right):=\int_{A} P_{\theta}\left(x_{t+1} \mid x^{t}\right) d \mu\left(. \mid x^{t}\right):=\int_{A} P_{\theta}\left(x_{t+1} \mid x^{t}\right) \frac{P_{\theta}\left(x^{t}\right) d \mu}{\int_{\Theta} P_{\theta}\left(x^{t}\right) d \mu} .
$$

When $A=\Theta$ we use the lighter notation $P^{\mu}\left(x \mid x^{t}\right):=P^{\mu}\left(\Theta \times x_{t+1} \mid x^{t}\right)$.

\section{Long-run ambiguity}

As in (Marinacci, 2002), we consider the difference between a decision-maker's expected utility under the most advantageous prior and under the least advantageous prior in $\mathcal{C}$ to be a measure

\footnotetext{
${ }^{4}$ We refer the reader to Nielsen and Garcia (2009) for a brief and schematic description of the main characteristic of the exponential family and a useful mapping between their canonical and natural parametrization.

${ }^{5}$ We rule out the possibility of observing an event which is impossible according to all models in $\mathcal{M}$.
} 
of the ambiguity that the decision-maker perceives in evaluating an act. If the set of priors $\mathcal{C}$ is compact, as we always assume, a tight sufficient condition for this difference to be zero is that the posteriors calculated from all priors in $\mathcal{C}$ eventually coincide (Marinacci and Massari, 2019).

Definition 2. Ambiguity fades away at path $x^{\infty} \in X^{\infty}$ if,

$$
\lim _{t \rightarrow \infty}\left[\sup _{\mu^{\prime}, \mu^{\prime \prime} \in \mathcal{C}} \int_{X}\left|d P^{\mu^{\prime \prime}}\left(x \mid x^{t}\right)-d P^{\mu^{\prime}}\left(x \mid x^{t}\right)\right|\right]=0
$$

where, for each $t>0, x^{t}$ indicates the first $t$ realizations of path $x^{\infty}$.

Definition 2 does not depend on the true model, which in any practical learning situation is not known by the decision-maker. It requires that all priors concentrate eventually on the same parameter (or on a set of parameters with identical predictions) on the realized path. Its relation with the familiar notion of weak merging (Kalai and Lehrer, 1994) is as follows. In well-specified learning problems all priors weakly merge to the true and ambiguity fades away. However, ambiguity might and does fade away in many misspecified learning problems in which the priors do not weakly merge with the truth.

\section{Main result}

In this section, we identify conditions that guarantee that ambiguity fades away in the long-run when $\Theta$ has positive Lebesgue measure. These regularity conditions are borrowed from Grünwald (2007) conditions for the BIC approximation (Schwarz (1978), Clarke and Barron (1990)), to which we add a compactness assumption on the set of priors $\mathcal{C}$ to ensure convergence.

Definition 3. The learning problem is regular if

A1: $\mathcal{M}$ is a regular exponential family;

A2: the set of priors, $\mathcal{C}$, is compact;

A3: priors in $\mathcal{C}$ are continuous and strictly positive on every compact subset of $\Theta$.

Condition $\mathbf{A 1}$ is a high order assumption that limits our attention to densities that are measurable jointly in $x$ and $\theta$ and regular enough for the empirical maximum likelihood to be unique in every period (in the canonical representation $\Theta$ is a convex set). Further, it allows writing the Fisher information matrix as the Hessian of the relative entropy. This assumption is stronger than condition $i$ ) of Berk (1966) and, together with A3, allows us to drop all assumptions about the data generating process. Unlike Berk (1966), we do not require draws to be iid. A2 is needed to ensure uniform convergence in the set of priors (Marinacci, 2002). A3 requires that priors have full, and thus common, support. This assumption reflects the attitude 
of an agent that does not rule out a-priori any parameter choice. The restriction on priors to be strictly positive in every compact subset of $\Theta$, rather than $\Theta$ itself, is due to the fact that $|\Theta|=\mathbb{R}^{k}$ for many members of the exponential family in the the canonical representation.

Definition 4. $\hat{\theta}\left(x^{t}\right)$ denotes the (vector valued) maximum likelihood estimator at $x^{t}$ :

$$
\hat{\theta}\left(x^{t}\right)=\underset{\theta \in \Theta}{\operatorname{argmax}} P_{\theta}\left(x^{t}\right)
$$

The equality in the definition above is justified because Assumptions A1 and A3 guarantee that the support, $\Theta$, is convex, so that a unique maximum likelihood exists at every finite history. We now present our main result: ambiguity fades away on all sequences such that the sequence of maximum likelihoods is bounded.

\section{Theorem 1.}

If the learning problem is regular, ambiguity fades away on all sequences such that lim sup $\left\|\hat{\theta}_{t}\right\|<$ $\infty$.

Proof. See Appendix.

Theorem 1 makes no reference to the truth. The point of view we adopt in Theorem 1 is empirical and differs from that of standard convergence results (e.g., Blackwell and Dubins (1962); Doob (1949); Berk (1966)). Instead of postulating the existence of a true distribution and deriving almost sure results, we show that convergence to the same predictive distributions occurs on all paths in which the sequence of maximum likelihood parameters is uniformly bounded. Being agnostic about the true distribution renders our approach particularly suited to discuss convergence in possibly misspecified learning environments.

Theorem 1 shows that ambiguity cannot persist in the long-run in the multiple-priors Bayesian learning model with naturally rich support. Its result naturally translates to exponential families when expressed in their canonical parameterization. For example, it tells us that:

- Ambiguity fades away on all sequences with frequency of heads uniformly bounded away from 0 and 1 if the Bayesian decision-maker believes draws are from an ambiguous coin and $\mathcal{C}$ is a compact set of not-degenerate Beta priors on the probability of heads $p^{h}$ (i.e., $\mathcal{C}=\{\operatorname{Beta}(\alpha, \beta), \alpha \in[a, b], \beta \in[c, d]\}$, with $[a, b],[c, d]$ strictly positive, finite intervals). In order to apply Theorem 1, we need to verify that on all sequences with frequency of heads $p^{h}$ uniformly bounded away from 0 and 1 the maximum likelihood estimator of the natural parameter of Bernoulli family $\hat{\theta}$ satisfies the condition limsup $\left\|\hat{\theta}_{t}\right\|<\infty$. From the 
conversion table of Nielsen and Garcia (2009), we see that $\theta^{p_{h}}=\ln \frac{p_{h}}{1-p_{h}}$; so, $\left(\left\|\hat{\theta}_{t}^{p_{h}}\right\|\right)_{t=1}^{\infty}$ is bounded if and only if $\left(\hat{p}_{t}^{h}\right)_{t=1}^{\infty}$ is uniformly bounded away from 0 and 1 .

- Ambiguity fades away on all bounded sequences, if the Bayesian decision-maker believes that realisations are Gaussian with known positive variance $\sigma$ and unknown mean $\mu$, and he has a compact set of non-degenerate Gaussian priors on the values of $\mu$ (i.e., $\mathcal{C}_{\mu}:=$ $\left\{\mathcal{N}\left(\mu^{\mu^{*}}, \sigma^{\mu^{*}}\right), \mu^{\mu} \in[a, b], \sigma^{\mu} \in[c, d]\right\}$ with $[a, b]$ finite and $[c, d]$ finite strictly positive intervals). In order to apply Theorem 1, we need to verify that if the sequence is bounded, the maximum likelihood estimator of the natural parameters of the Gaussian family $\hat{\theta}=$ $\left(\hat{\theta}^{1}, \hat{\theta}^{2}\right) \in \mathbb{R} \times\left(R^{-}\right)$satisfies the condition limsup $\left\|\hat{\theta}_{t}\right\|<\infty$. From the conversion table of Nielsen and Garcia (2009), we see that $\theta=\left(\frac{\mu}{\sigma^{2}},-\frac{1}{2 \sigma^{2}}\right)$, so that $\hat{\theta}=\left(\frac{\hat{\mu}_{t}}{\hat{\sigma}_{t}^{2}},-\frac{1}{2 \hat{\sigma}_{t}^{2}}\right)$, where $\hat{\mu}_{t}$ and $\hat{\sigma}_{t}$ are the maximum likelihood estimators of mean, $\hat{\mu}_{t}:=\frac{1}{t} \sum_{\tau=1}^{t} x_{t}$, and variance, $\hat{\sigma}_{t}^{2}:=\frac{1}{t} \sum_{\tau=1}^{t}\left(x_{\tau}-\hat{\mu}_{t}\right)$. So, limsup $\left\|\hat{\theta}_{t}\right\|<\infty$ because for all $t, \hat{\mu}_{t}<\infty$ on all bounded sequences and $\sigma>0 \Rightarrow \hat{\sigma}_{t}>0$ for all large $t$.

\section{Appendix}

In this appendix $\hat{\theta}_{t}:=\hat{\theta}\left(x^{t}\right)$, and we make use of the K-L divergence. Let $S(\mathcal{M}, \Theta)$ be the set of sequences such that limsup $\left\|\hat{\theta}_{t}\right\|<\infty$.

Definition 5. The K-L divergence from $P_{\hat{\theta}_{t}}$ to $P_{\theta}$ is

$$
D\left(P_{\hat{\theta}_{t}} \| P_{\theta}\right):=E_{P_{\hat{\theta}_{t}}}\left[\ln \frac{P_{\hat{\theta}_{t}}(x)}{P_{\theta}(x)}\right] .
$$

The proof is a standard application of the Laplace method. The strategy is to show that for $t$ large, for all priors in $\mathcal{C}$, the value of the integral of the unconditional probabilities is well approximated by the value it assumes on a shrinking interval around the minimizer of the K-L divergence (i.e., by the maximum likelihood model). Because $\Theta$ is convex and $-D\left(P_{\hat{\theta}_{t}} \| P_{\theta}\right)$ is strictly concave, this minimizer is unique. Because the exponential family is regular $x^{\infty} \in S(\mathcal{M}, \Theta) \Rightarrow\left(\hat{\theta}_{t}\right)_{t=1}^{\infty}$ belongs to a compact subset of $\Theta$ and the approximation below is never on the boundary of the support. ${ }^{6}$

\section{Proof of Theorem 1}

Proof. $\mathcal{C}$ compact $\Rightarrow \forall x^{t}, \operatorname{argmax}_{\mu \in \mathcal{C}} \lim _{t \rightarrow \infty} \int_{X}\left|d P^{g}\left(x \mid x^{t}\right)-d P^{h}\left(x \mid x^{t}\right)\right|$ exists. Thus, it suffices to show that if the learning problem is regular, then $\forall x^{\infty} \in \hat{S}(\mathcal{M}, \Theta)$ and $\forall g, h \in \mathcal{C}, \lim _{t \rightarrow \infty} \int_{X} \mid d P^{g}\left(x \mid x^{t}\right)-$ $d P^{h}\left(x \mid x^{t}\right) \mid=0$.

\footnotetext{
${ }^{6}$ For non-regular member of the exponential family, the proof below cannot be adopted for those sequences on which the maximum likelihood estimator are within an order $1 / \sqrt{t}$ to the boundary of $\theta$ because Laplace approximation is truncated. For those sequences the shape of $P_{\theta}\left(x^{t}\right)$ becomes a truncated Gaussian with a reduced value of the integral in Lemma 1. For those sequences, however, the discrepancy in the approximation is only a constant (Xie and Barron, 2000), and it would not affect our result.
} 


$$
\begin{aligned}
& 0 \leq \lim _{t \rightarrow \infty} \int_{X}\left|d P^{g}\left(x \mid x^{t}\right)-d P^{h}\left(x \mid x^{t}\right)\right|:=\lim _{t \rightarrow \infty} \int_{X}\left|\int_{\Theta} P_{\theta}(x)\left(\frac{P_{\theta}\left(x^{t}\right) g(\theta)}{P^{g}\left(x^{t}\right)}-\frac{P_{\theta}\left(x^{t}\right) h(\theta)}{P^{h}\left(x^{t}\right)}\right) d \theta\right| d x \\
& ={ }^{a} \lim _{t \rightarrow \infty} \int_{X}\left|\int_{\Theta} P_{\theta}(x)\left(\frac{e^{-t D\left(P_{\hat{\theta}_{t}} \| P_{\theta}\right)} g(\theta)}{\int_{\Theta} e^{-t D\left(P_{\hat{\theta}_{t}} \| P_{\theta}\right)} g(\theta) d \theta} \frac{P_{\hat{\theta}_{t}}\left(x^{t}\right)}{P_{\hat{\theta}_{t}}\left(x^{t}\right)}-\frac{e^{-t D\left(P_{\hat{\theta}_{t}} \| P_{\theta}\right)} h(\theta)}{\int_{\Theta} e^{-t D\left(P_{\hat{\theta}_{t}} \| P_{\theta}\right)} h(\theta) d \theta} \frac{P_{\hat{\theta}_{t}}\left(x^{t}\right)}{P_{\hat{\theta}_{t}}\left(x^{t}\right)}\right) d \theta\right| d x \\
& ={ }^{b} \int_{X} \lim _{t \rightarrow \infty}\left|\int_{\Theta} P_{\theta}(x)\left(\frac{e^{-t D\left(P_{\hat{\theta}_{t}} \| P_{\theta}\right)} g(\theta)}{\int_{\Theta} e^{-t D\left(P_{\hat{\theta}_{t}} \| P_{\theta}\right)} g(\theta) d \theta}-\frac{e^{-t D\left(P_{\hat{\theta}_{t}} \| P_{\theta}\right)} h(\theta)}{\int_{\Theta} e^{-t D\left(P_{\hat{\theta}_{t}} \| P_{\theta}\right)} h(\theta) d \theta}\right) d \theta\right| d x \\
& ={ }^{c, d} \int_{X} \lim _{t \rightarrow \infty}\left|\int_{B_{t}} P_{\theta}(x)\left(\frac{e^{-t D\left(P_{\hat{\theta}_{t}} \| P_{\theta}\right)} g(\theta)}{\int_{B_{t}} e^{-t D\left(P_{\hat{\theta}_{t}} \| P_{\theta}\right)} g(\theta) d \theta}-\frac{e^{-t D\left(P_{\hat{\theta}_{t}} \| P_{\theta}\right)} h(\theta)}{\int_{B_{t}} e^{-t D\left(P_{\hat{\theta}_{t}} \| P_{\theta}\right)} h(\theta) d \theta}\right) d \theta\right| d x \\
& \leq^{e} \quad \int_{X} \lim _{t \rightarrow \infty}\left|\int_{B_{t}} P_{\theta}(x) \max \left\{\left|\frac{\frac{\sqrt{(2 \pi)^{k}} g_{t}^{+}}{\sqrt{t^{k} \operatorname{det}\left(I_{t}^{-}\right)}}}{\frac{\sqrt{(2 \pi)^{k}} g_{t}^{-}}{\sqrt{t^{k} \operatorname{det}\left(I_{t}^{+}\right)}}}-\frac{\frac{\sqrt{(2 \pi)^{k}} h_{t}^{-}}{\sqrt{t^{k} \operatorname{det}\left(I_{t}^{+}\right)}}}{\frac{\left.\sqrt{(2 \pi)^{k}} h_{t}^{+}\right)}{\sqrt{t^{k} \operatorname{det}\left(I_{t}^{-}\right)}}}\right| ;\left|\frac{\frac{\sqrt{(2 \pi)^{k}} g_{t}^{-}}{\sqrt{t^{k} \operatorname{det}\left(I_{t}^{+}\right)}}}{\sqrt{(2 \pi)^{k}} g_{t}^{+}}-\frac{\frac{\sqrt{(2 \pi)^{k}} h_{t}^{+}}{\sqrt{t^{k} \operatorname{det}\left(I_{t}^{-}\right)}}}{\frac{\sqrt{t^{k} \operatorname{det}\left(I_{t}^{-}\right)}}{\sqrt{t^{k} \operatorname{det}\left(I_{t}^{+}\right)}}}\right|\right\}\right| d x
\end{aligned}
$$

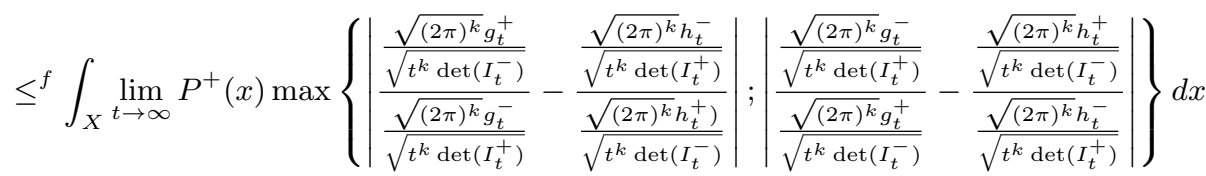

$$
\begin{aligned}
& =\int_{X} \lim _{t \rightarrow \infty} P^{+}(x) \max \left\{\left|\frac{g_{t}^{+}}{g_{t}^{-}} \frac{\sqrt{\operatorname{det}\left(I_{t}^{+}\right)}}{\sqrt{\operatorname{det}\left(I_{t}^{-}\right)}}-\frac{h_{t}^{-}}{h_{t}^{+}} \frac{\sqrt{\operatorname{det}\left(I_{t}^{-}\right)}}{\sqrt{\operatorname{det}\left(I_{t}^{+}\right)}}\right| ;\left|\frac{g_{t}^{-}}{g_{t}^{+}} \frac{\sqrt{\operatorname{det}\left(I_{t}^{-}\right)}}{\sqrt{\operatorname{det}\left(I_{t}^{+}\right)}}-\frac{h_{t}^{+}}{h_{t}^{-}} \frac{\sqrt{\operatorname{det}\left(I_{t}^{+}\right)}}{\sqrt{\operatorname{det}\left(I_{t}^{-}\right)}}\right| ;\right\} d x \\
& ={ }^{g} 0 .
\end{aligned}
$$

a) A known result for members of the exponential family (e.g., Grünwald, 2007, Chapter 8) is that

$$
P^{g}\left(x^{t}\right)=\int_{\Theta} P_{\theta}\left(x^{t}\right) g(\theta) d \theta=\frac{\int_{\Theta} e^{-t D\left(P_{\hat{\theta}_{t}} \| P_{\theta}\right)} g(\theta) d \theta}{P_{\hat{\theta}_{t}}\left(x^{t}\right)} .
$$

b) We can exchange the order of limit and integration by the Lebesgue dominated convergence theorem.

c) $B_{t}$ is a neighbourhood of the maximum likelihood that, in all dimensions, converges to zero at a rate slightly slower than $\sqrt{\frac{1}{t}}$. That is $B_{t}:=\left\{\theta \in \Theta \subset \mathbb{R}^{k}: \forall i=1, \ldots, k,\left|\theta^{i}-\hat{\theta}^{i}\right| \leq t^{-\frac{1}{2}-\alpha}\right\}$ for some $\alpha \in(0, .5)$.

d) By Lemma 1 (i), $\int_{\Theta \backslash B_{t}} e^{-t D\left(P_{\hat{\theta}_{t}} \| P_{\theta}\right)} h(\theta) d \theta \rightarrow 0$ exponentially fast and it can be ignored in the calculation of the limit.

e) By Lemma 1 (ii), with $I:=E_{P_{\hat{\theta}_{t}}}\left[-\frac{\partial^{2}}{\partial \theta_{i} \partial \theta_{j}} \ln P_{\theta}\right]_{\theta=\hat{\theta}_{t}}, \operatorname{det}\left(I_{t}^{-}\right)=\inf _{\theta^{\prime} \in B_{t}} \operatorname{det}\left(I\left(\theta^{\prime}\right)\right), \operatorname{det}\left(I_{t}^{+}\right)=$ $\sup _{\theta^{\prime} \in B_{t}} \operatorname{det}\left(I\left(\theta^{\prime}\right)\right), g_{t}^{-}=\inf _{\theta^{\prime} \in B_{t}} g\left(\theta^{\prime}\right), g_{t}^{+}=\sup _{\theta^{\prime} \in B_{t}} g\left(\theta^{\prime}\right), h_{t}^{-}=\inf _{\theta^{\prime} \in B_{t}} h\left(\theta^{\prime}\right), h_{t}^{+}=\sup _{\theta^{\prime} \in B_{t}} h\left(\theta^{\prime}\right)$.

f) With $P^{+}(x)=\sup _{\theta \in B_{t}} P_{\theta}(x)<1$.

g) Continuity and strict positivity of $g(\cdot), h(\cdot)$ in $\operatorname{det}(I(\cdot))$ in $B_{t}$ guarantee that for all $x^{\infty} \in \hat{S}(\mathcal{M}, \Theta)$ the following limit holds: ${ }^{7}$

$$
\max \left\{\left|\frac{g_{t}^{+}}{g_{t}^{-}} \frac{\sqrt{\operatorname{det}\left(I_{t}^{+}\right)}}{\sqrt{\operatorname{det}\left(I_{t}^{-}\right)}}-\frac{h_{t}^{-}}{h_{t}^{+}} \frac{\sqrt{\operatorname{det}\left(I_{t}^{-}\right)}}{\sqrt{\operatorname{det}\left(I_{t}^{+}\right)}}\right| ;\left|\frac{g_{t}^{-}}{g_{t}^{+}} \frac{\sqrt{\operatorname{det}\left(I_{t}^{-}\right)}}{\sqrt{\operatorname{det}\left(I_{t}^{+}\right)}}-\frac{h_{t}^{+}}{h_{t}^{-}} \frac{\sqrt{\operatorname{det}\left(I_{t}^{+}\right)}}{\sqrt{\operatorname{det}\left(I_{t}^{-}\right)}}\right|\right\} \rightarrow 0 .
$$

\footnotetext{
${ }^{7}$ By construction, for all $\forall x^{\infty} \in \hat{S}(\mathcal{M}, \Theta), B_{t}$ is a subset of a compact of $\Theta$; thus, by $(\mathbf{A} \mathbf{3}), g(\cdot)$ and $h(\cdot)$ are continuous and bounded away from zero. $\operatorname{det}(I(\cdot))$ is continuous bounded away from zero because $\mathcal{M}$ is a member of the exponential family (A1).
} 
Lemma 1. Let $\mathcal{M}$ be a regular member of the exponential family parameterized by $\Theta$ and $\mu$ a prior that satisfies $\boldsymbol{A} 3$, then, $\forall x^{\infty} \in \hat{S}(\mathcal{M}, \Theta)$,

$$
\int_{\Theta} e^{-t D\left(P_{\hat{\theta}_{t}} \| P_{\theta}\right)} \mu(\theta) d \theta=\int_{\Theta \backslash B_{t}} e^{-t D\left(P_{\hat{\theta}_{t}} \| P_{\theta}\right)} \mu(\theta) d \theta+\int_{B_{t}} e^{-t D\left(P_{\hat{\theta}_{t}} \| P_{\theta}\right)} \mu(\theta) d \theta,
$$

and, for $t$ large, the following bounds holds uniformly when $B_{t}$ is a neighbourhood of the maximum likelihood such that $\operatorname{diam}\left(B_{t}\right) \rightarrow^{t \rightarrow \infty} 0$ at a rate slightly slower than $\sqrt{\frac{1}{t}}$.

(i) First integral: $\exists k>0: \mathcal{I}_{1}=\int_{\Theta \backslash B_{t}} e^{-t D\left(P_{\hat{\theta}_{t}} \| P_{\theta}\right)} \mu(\theta) d \theta<e^{-r t^{2 \alpha}}$.

(ii) Second integral: Let $\mathcal{I}_{2}=\int_{B_{t}} e^{-t D\left(P_{\hat{\theta}_{t}} \| P_{\theta}\right)} \mu(\theta) d \theta ; I\left(\theta_{t}\right):=E_{P_{\hat{\theta}_{t}}}\left\{-\frac{\partial^{2}}{\partial \theta_{i} \partial \theta_{j}} \ln P_{\theta}\right\}_{\theta=\hat{\theta}_{t}}$ be the Fisher information evaluated at the maximum likelihood parameter, ${ }^{8} k$ be the dimensionality of $\Theta$, $\operatorname{det}\left(I_{t}^{-}\right)=\inf _{\theta^{\prime} \in B_{t}} \operatorname{det}\left(I\left(\theta^{\prime}\right)\right), \operatorname{det}\left(I_{t}^{+}\right)=\sup _{\theta^{\prime} \in B_{t}} \operatorname{det}\left(I\left(\theta^{\prime}\right)\right)$; and $\mu_{t}^{-}=\inf _{\theta^{\prime} \in B_{t}} \mu\left(\theta^{\prime}\right), \mu_{t}^{+}=$ $\sup _{\theta^{\prime} \in B_{t}} \mu\left(\theta^{\prime}\right)$, then

$$
\frac{\mu_{t}^{-}(2 \pi)^{k / 2}}{\sqrt{t^{k} \operatorname{det}\left(I_{t}^{+}\right)} i} \leq \mathcal{I}_{2} \leq \frac{\mu_{t}^{+}(2 \pi)^{k / 2}}{\sqrt{t^{k} \operatorname{det}\left(I_{t}^{-}\right)}} .
$$

Proof. Let $B_{t}:=\left\{\theta \in \Theta \subset \mathbb{R}^{k}: \forall i=1, \ldots, k,\left|\theta^{i}-\hat{\theta}^{i}\right| \leq t^{-\frac{1}{2}-\alpha}\right\}$ for some $\alpha \in(0, .5)$. To gain intuition, take $\alpha$ very small, so that $B_{t}$ is a neighborhood of the maximum likelihood that shrinks to 0 at a rate slightly slower than $1 / \sqrt{t}$ in all dimensions. Because $x^{\infty} \in \hat{S}(\mathcal{M}, \Theta)$ and $\mu$ is continuous and positive on all compact subsets of $\Theta$ (by A3), there is a $T: \forall t>T, B_{t} \subset \hat{\Theta}$ where $\hat{\Theta}$ is a compact subset of $\Theta$ in which $\mu>\epsilon>0$ for some positive $\epsilon$. We always assume $t>T$.

The proof is done by performing a second-order Taylor expansion of $\left.D\left(P_{\hat{\theta}_{t}}\right) \| P_{\theta}\right)$ to bound the two integrals. $\mathcal{M}$ is an exponential family; thus, $D\left(P_{\hat{\theta}_{t}} \| P_{\theta}\right)$ can be exactly approximated in $B_{t}$ as follows (see Grünwald, 2007, chapter 19):

$$
D\left(P_{\hat{\theta}_{t}} \| P_{\theta}\right)=\frac{1}{2}\left(\hat{\theta}_{t}-\theta\right)^{T} I\left(\theta^{*}\right)\left(\hat{\theta}_{t}-\theta\right),
$$

for some $\theta^{*} \in B_{t}$ such that $\theta^{*}$ lies between $\theta$ and $\hat{\theta}_{t}-$ here, $I:=E_{P_{\hat{\theta}_{t}}}\left[-\frac{\partial^{2}}{\partial \theta_{i} \partial \theta_{j}} \ln P_{\theta}\right]_{\theta=\hat{\theta}_{t}}$; because $\mathcal{M}$ is an exponential family, this is the Fisher information matrix evaluated at the maximum likelihood estimator.

(i) First integral: Because $D\left(P_{\hat{\theta}_{t}} \| P_{\theta}\right)$, as a function of $\theta$, is strictly convex, has a minimum at $\theta=\hat{\theta}_{t}$, and is increasing in $\left\|\theta-\hat{\theta}_{t}\right\|$, the following holds:

$$
0<\int_{\Theta \backslash B_{t}} e^{-t D\left(P_{\hat{\theta}_{t}} \| P_{\theta}\right)} g(\theta) d \theta<\int_{\Theta \backslash B_{t}} e^{-t \min _{\theta \in \Theta \backslash B_{t}} D\left(P_{\hat{\theta}_{t}} \| P_{\theta}\right)} g(\theta) d \theta
$$

where

$$
\min _{\theta \in \Theta \backslash B_{t}} D\left(P_{\hat{\theta}_{t}} \| P_{\theta}\right)={ }^{(a)} \min _{\theta \in \partial B_{t}} D\left(P_{\hat{\theta}_{t}} \| P_{\theta}\right) \geq{ }^{(b)} \frac{1}{2} t^{-1+2 \alpha} \min _{\theta \in \operatorname{int}(\Theta)} \mathbf{1}^{T} I(\theta) \mathbf{1},
$$

where $(a)$ holds because strict convexity of $D(\cdot \| \cdot)$ implies that that the $D(\cdot \| \cdot)$ is minimal at the boundary of $B_{t}$; and $(b)$ holds, with 1 being a $k$-dimensional vector of $1 \mathrm{~s}$, because of the

${ }^{8}$ Which is positive definite because $\mathcal{M}$ is an exponential family. 
definition of $B_{t}$ and Equation 2. So, since $I(\theta)$ is continuous and $>0$ for all $\theta \in \Theta$, and also $\int_{\Theta \backslash B_{t}} \mu(\theta) d \theta \leq 1$

$$
0<\int_{\Theta \backslash B_{t}} e^{-t D\left(P_{\hat{\theta}_{t}} \| P_{\theta}\right)} g(\theta) d \theta<\int_{\Theta \backslash B_{t}} e^{-t\left(\frac{1}{2} t^{-1+2 \alpha} \min _{\theta \in i n t(\Theta)} I(\theta)\right)} g(\theta) d \theta<e^{-r t^{2 \alpha}},
$$

for $r=\frac{1}{2} \min _{\theta \in \operatorname{int}(\Theta)} I(\theta)>0$.

(ii) Second integral: by Equation 2,

$$
\mathcal{I}_{2}=\int_{B_{t}} e^{-t D\left(P_{\hat{\theta}_{t}} \| P_{\theta}\right)} g(\theta) d \theta=\int_{B_{t}} e^{-\frac{t}{2}\left(\hat{\theta}_{t}-\theta\right)^{T} I\left(\theta^{\prime}\right)\left(\hat{\theta}_{t}-\theta\right)} g(\theta) d \theta
$$

where $\theta^{\prime}$ depends on $\theta$. Let $I_{t}^{-}=\underset{\theta^{\prime} \in B_{t}}{\operatorname{argmin}} \operatorname{det}\left(I\left(\theta^{\prime}\right)\right)$ and $I_{t}^{+}=\underset{\theta^{\prime} \in B_{t}}{\operatorname{argmax}} \operatorname{det}\left(I\left(\theta^{\prime}\right)\right)$; it follows that

$$
g_{t}^{-} \int_{B_{t}} e^{-\frac{t}{2}\left(\hat{\theta}_{t}-\theta\right)^{T} I_{t}^{+}\left(\hat{\theta}_{t}-\theta\right)} d \theta \leq \mathcal{I}_{2} \leq g_{t}^{+} \int_{B_{t}} e^{-\frac{t}{2}\left(\hat{\theta}_{t}-\theta\right)^{T} I_{t}^{-}\left(\hat{\theta}_{t}-\theta\right)} d \theta .
$$

Performing the substitutions $z^{T}=\left(\sqrt{t}\left(\hat{\theta}_{t}-\theta\right)\right)^{T} A_{t}^{+}$on the left integral and $z^{T}=\left(\sqrt{t}\left(\hat{\theta}_{t}-\theta\right)\right)^{T} A_{t}^{-}$ on the right integral - where $A_{t}^{+}$and $A_{t}^{-}$are matrixes such that $A_{t}^{+}\left(A_{t}^{+}\right)^{T}=I_{t}^{+}$and $A_{t}^{-}\left(A_{t}^{-}\right)^{T}=$ $I_{t}^{-}$, respectively -, we get

$$
\frac{g_{t}^{-}}{\sqrt{t^{k} \operatorname{det}\left(I_{t}^{+}\right)}} \int_{|z|<\left|t^{\alpha} \mathbf{1}^{T} A_{t}^{+}\right|} e^{-\frac{1}{2} z^{T} z} d z \leq \mathcal{I}_{2} \leq \frac{g_{t}^{+}}{\sqrt{t^{k} \operatorname{det}\left(I_{t}^{-}\right)}} \int_{|z|<\left|t^{\alpha} \mathbf{1}^{T} A_{t}^{-}\right|} e^{-\frac{1}{2} z^{T} z} d z,
$$

- where for a vector $x$, the vector $|x|$ indicates the vector whose entries are the absolute values of $x$ and $k$ is the dimensionality of $\Theta^{k}$ - and recognize these integrals as proportional to standard mutivariate Gaussian. Because, as $t \rightarrow \infty, I_{t}^{-} \rightarrow I\left(\hat{\theta}_{t}\right)$ and $I_{t}^{+} \rightarrow I\left(\hat{\theta}_{t}\right)$, the domain of integration tends to infinity for both integrals, they both converge to $\sqrt{(2 \pi)^{k}}$.

This approximation holds uniformly for all $x^{\infty} \in \hat{S}(\mathcal{M}, \Theta)$ because $i$ ) the bound on $\mathcal{I}_{1}$ does not depend on $x^{t}$, and $i i$ ) convergence of $\mathcal{I}_{2}$ is uniform because $\mathbf{A} \mathbf{1}$ and $\mathbf{A} \mathbf{3}$ guarantee that $g(\theta)$ and $I(\theta)$ are continuous, positive functions of $\theta$ over every compact subset of $\Theta$.

\section{References}

Augustin, P. and Izhakian, Y. (2020). Ambiguity, volatility, and credit risk. The Review of Financial Studies, 33(4):1618-1672.

Berger, J. O. (2013). Statistical decision theory and Bayesian analysis. Springer Science \& Business Media.

Berk, R. H. (1966). Limiting behavior of posterior distributions when the model is incorrect. The Annals of Mathematical Statistics, 37(1):51-58.

Blackwell, D. and Dubins, L. (1962). Merging of opinions with increasing information. The Annals of Mathematical Statistics, 33(3):882-886.

Clarke, B. S. and Barron, A. R. (1990). Information-theoretic asymptotics of bayes methods. Information Theory, IEEE Transactions on, 36(3):453-471. 
Doob, J. L. (1949). Application of the theory of martingales. Colloques Internationaux du Centre National de la Recherche Scientifique Paris.

Epstein, L. G. and Schneider, M. (2007). Learning under ambiguity. The Review of Economic Studies, 74(4):1275-1303.

Garlappi, L., Uppal, R., and Wang, T. (2006). Portfolio selection with parameter and model uncertainty: A multi-prior approach. The Review of Financial Studies, 20(1):41-81.

Gilboa, I. and Marinacci, M. (2016). Ambiguity and the bayesian paradigm. In Readings in formal epistemology, pages 385-439. Springer.

Grünwald, P. D. (2007). The minimum description length principle. MIT press.

Hansen, L. P., Sargent, T. J., and Tallarini Jr, T. D. (1999). Robust permanent income and pricing. Review of Economic studies, pages 873-907.

Kajii, A. and Ui, T. (2006). Agreeable bets with multiple priors. Journal of Economic Theory, 128(1):299-305.

Kalai, E. and Lehrer, E. (1994). Weak and strong merging of opinions. Journal of Mathematical Economics, 23(1):73-86.

Marinacci, M. (2002). Learning from ambiguous urns. Statistical Papers, 43(1):143-151.

Marinacci, M. and Massari, F. (2019). Learning from ambiguous and misspecified models. Journal of Mathematical Economics, 84:144-149.

Markowitz, H. (1952). Portfolio selection. The journal of finance, 7(1):77-91.

Nielsen, F. and Garcia, V. (2009). Statistical exponential families: A digest with flash cards. arXiv preprint arXiv:0911.4863.

Nishimura, K. G. and Ozaki, H. (2004). Search and knightian uncertainty. Journal of Economic Theory, 119(2):299-333.

Schwarz, G. (1978). Estimating the dimension of a model. The Annals of Statistics, 6(2):461464.

Sharpe, W. F. (1970). Portfolio theory and capital markets, volume 217. McGraw-Hill New York.

Werner, J. (2019). Speculative trade under ambiguity. Technical report, mimeo.

Xie, Q. and Barron, A. R. (2000). Asymptotic minimax regret for data compression, gambling, and prediction. IEEE Transactions on Information Theory, 46(2):431-445. 